\title{
Doxorubicin-mediated radiosensitivity in multicellular spheroids from a lung cancer cell line is enhanced by composite micelle encapsulation
}

This article was published in the following Dove Press journal:

International Journal of Nanomedicine

27 May 2012

Number of times this article has been viewed

\author{
Wen-Hong $\mathrm{Xu}^{\prime}$ \\ Min $\mathrm{Han}^{2}$ \\ Qi Dong ${ }^{3}$ \\ Zhi-Xuan Fu ${ }^{3}$ \\ Yuan-Yuan Diao ${ }^{2}$ \\ Hai Liu ${ }^{3}$ \\ Jing $X u^{3}$ \\ Hong-Liang Jiang ${ }^{4}$ \\ Su-Zhan Zhang ${ }^{3}$ \\ Shu Zheng ${ }^{3}$ \\ Jian-Qing $\mathrm{Gao}^{2}$ \\ Qi-Chun Wei'
}

'Department of Radiation Oncology, The Second Affiliated Hospital, School of Medicine, Zhejiang University, ${ }^{2}$ Institute of Pharmaceutics, College of Pharmaceutical Sciences, ${ }^{3}$ Cancer Institute (Key Laboratory of Cancer Prevention and Intervention, China National Ministry of Education), The Second Affiliated Hospital, School of Medicine, ${ }^{4}$ Department of Polymer Science and Engineering, Zhejiang University, Hangzhou, Zhejiang, China
Background: The purpose of this study is to evaluate the efficacy of composite doxorubicinloaded micelles for enhancing doxorubicin radiosensitivity in multicellular spheroids from a non-small cell lung cancer cell line.

Methods: A novel composite doxorubicin-loaded micelle consisting of polyethylene glycolpolycaprolactone/Pluronic P105 was developed, and carrier-mediated doxorubicin accumulation and release from multicellular spheroids was evaluated. We used confocal laser scanning microscopy and flow cytometry to study the accumulation and efflux of doxorubicin from A549 multicellular spheroids. Doxorubicin radiosensitization and the combined effects of irradiation and doxorubicin on cell migration and proliferation were compared for the different doxorubicin delivery systems.

Results: Confocal laser scanning microscopy and quantitative flow cytometry studies both verified that, for equivalent doxorubicin concentrations, composite doxorubicin-loaded micelles significantly enhanced cellular doxorubicin accumulation and inhibited doxorubicin release. Colony-forming assays demonstrated that composite doxorubicin-loaded micelles are radiosensitive, as shown by significantly reduced survival of cells treated by radiation + composite micelles compared with those treated with radiation + free doxorubicin or radiation alone. The multicellular spheroid migration area and growth ability verified higher radiosensitivity for the composite micelles loaded with doxorubicin than for free doxorubicin.

Conclusion: Our composite doxorubicin-loaded micelle was demonstrated to have radiosensitization. Doxorubicin loading in the composite micelles significantly increased its cellular uptake, improved drug retention, and enhanced its antitumor effect relative to free doxorubicin, thereby providing a novel approach for treatment of cancer.

Keywords: doxorubicin, lung cancer, micelles, radiosensitivity, spheroids

\section{Introduction}

Lung cancer is the most common primary malignant tumor in humans and is the leading cause of cancer mortality in the world. ${ }^{1}$ Its occurrence is increasing in both developing and developed countries, and in the latter, lung cancer is the second most frequently occurring cancer in both men and women. However, treatment for lung cancer remains relatively ineffective, and the five-year survival rate for advanced disease is only $15 \% .^{2}$

The 2011 National Comprehensive Cancer Network guidelines for lung cancer state that radiation has a potential role in the treatment of all stages of lung cancer, as either definitive treatment or as palliative therapy. It is recommended that radiation oncology should form part of a multidisciplinary evaluation provided for all patients
Correspondence: Qi-Chun Wei

Department of Radiation Oncology,

The Second Affiliated Hospital,

School of Medicine, Zhejiang University,

88 Jiefang Road, Hangzhou,

Zhejiang Province 310009 , China

Tel +86 57I 87783537

Fax +86 57I 87784404

Email weiqichun0I@yahoo.com.cn

Qichun_Wei@zju.edu.cn 
who may benefit from definitive local therapy, particularly those with absolute or relative contraindications to surgery as determined by a thoracic surgeon.

Although recent years have witnessed the development of radiation physics, biology, and radiotherapy facilities, the efficacy of radiation therapy is still not satisfactory. Research on radiosensitization, with the aim of improving the sensitivity of tumor cells to radiation, has been extensive. ${ }^{3-5}$ The ideal radiation sensitizer should significantly increase the efficacy of radiotherapy, but have little or no adverse effect on normal tissues. Antitumor drugs, such as doxorubicin, are widely used in cancer therapy. However, side effects, such as bone marrow suppression, heart toxicity, and mucositis, limit the use of cytotoxic antitumor drugs as radiosensitizers. To reduce the toxicity of these drugs and improve their therapeutic efficacy, various polymeric micelle systems have been designed as delivery vehicles. If these carriers could improve cellular uptake and retention of the delivered compound, radiosensitization might be achieved using smaller doses of chemotherapy drugs, thus lowering the risk of side effects. New drug carrier micelles have high stability and biocompatibility, via the enhanced permeability and retention effect of drugs towards tumor cells, with enhanced efficacy and reduced toxicity, and are capable of slow drug release. ${ }^{6-10}$ Hydrophobic drugs can be incorporated into the micelle by both chemical conjugation and physical entrapment. ${ }^{11}$

However, simple polymeric micelles may not provide a satisfactory effect. We have previously reported that the combination of polyethylene glycol (PEG), polycaprolactone (PCL), and Pluronic P105 significantly decreased cytotoxicity and intracellular accumulation of loaded doxorubicin in drug-resistant K562/ADR tumor cells. ${ }^{12}$ Moreover, we have previously discovered that composite micelles consisting of two kinds of polymers (PEG-PCL and P105), had a synergistic effect as drug carriers in overcoming or reversing multidrug resistance in drug-resistant tumor cells. ${ }^{13} \mathrm{We}$ observed that doxorubicin-loaded PEG-PCL/P105 composite micelles were more cytotoxic towards K562/ADR cells than were doxorubicin-loaded PEG-PCL or P105 single micelles or doxorubicin solution. ${ }^{13}$

Non-small cell lung cancer is more common than small cell lung cancer, occurring in $85 \%$ of patients with the disease. For non-small cell lung cancer, adenocarcinoma is particularly prone to metastasis, and therefore the human lung adenocarcinoma A549 cell line was selected to model non-small cell lung cancer in this study. Moreover, most studies of therapeutic efficacy in lung cancer cells have been carried out in monolayer cultures. ${ }^{14,15}$ However, the spheroid model is a three-dimensional cell culture system that more closely resembles in vivo tumor cell growth and is more physiologically relevant than monolayer cell growth. ${ }^{16,17}$ Individual tumor cells growing under these conditions face different microenvironments depending on their position inside the three-dimensional framework of the spheroid.

In this study, composite doxorubicin-loaded micelles consisting of PEG-PCL/Pluronic P105 were developed. A549 multicellular spheroids were incubated with drugloaded micelles and irradiated. Confocal laser scanning microscopy was used to measure the intensity of doxorubicin fluorescence and the depth of doxorubicin penetration into the spheroids, and flow cytometry was used to quantitate the doxorubicin fluorescence signal intensity, because doxorubicin is a fluorescent compound and cellular doxorubicin incorporation can be measured. Colony-forming assays were used to evaluate the radiosensitivity of composite doxorubicin-loaded micelles following ionizing radiation. We also studied the combined effects of irradiation and composite doxorubicin-loaded micelle treatment on tumor cell migration and proliferation in a well defined threedimensional spheroid culture system.

This study aimed to radiosensitize non-small cell lung cancer multicellular spheroids using doxorubicin-encapsulated micelles to achieve reduced toxicity and a higher therapeutic effect. Radiosensitization by increased intracellular accumulation and delayed drug release will facilitate screening for novel and effective radiosensitizers and development of improved radiosensitizer delivery in targeting systems.

\section{Materials and methods Reagents}

Fibroblast growth factor and epidermal growth factor were purchased from PeprotechAsia (Rocky Hill, NJ). B27 was purchased from Invitrogen Corporation (Camarillo, CA). RPMI 1640 medium, phosphate-buffered saline, fetal bovine serum, and $0.25 \%$ trypsin were purchased from GibcoBRL (Gaithersburg, MD). Doxorubicin hydrochloride was obtained from Zhejiang Hisun Pharmaceutical Co, Ltd. MPEGs and $\varepsilon$-caprolactone were purchased from SigmaAldrich (St Louis, MO). Pluronic P105 was a kind gift from the BASF Corporation (Ludwigshafen, Germany). Agarose was purchased from Bio Basic Inc (Markham, ON, Canada). MTT was from Shanghai Sangon Biological Engineering Technology and Service Co, Ltd (Shanghai, China). $\mathrm{CH}_{2} \mathrm{Cl}_{2}$, triethylamine, and other reagents were 
of analytical grade and supplied by Huadong Medical (Hangzhou, China).

\section{Cell line spheroids}

The human A549 cell line was obtained from the Cancer Institute, Second Affiliated Hospital of School of Medicine, Zhejiang University, cultured in RPMI 1640 medium supplemented with $10 \%$ fetal calf serum, $5000 \mathrm{U} / \mathrm{mL}$ penicillin, and $5 \mathrm{mg} / \mathrm{mL}$ streptomycin, and incubated at $37^{\circ} \mathrm{C}$ in a humidified atmosphere containing $5 \% \mathrm{CO}_{2}$. Spheroid A549 cultures were formed from exponentially growing monolayers. Briefly, cells were trypsinized, and $5 \times 10^{6}$ cells were then seeded in $50 \mathrm{~mL}$ of growth medium supplemented with epidermal growth factor $20 \mathrm{ng} / \mathrm{mL}$, basic fibroblast growth factor $10 \mathrm{ng} / \mathrm{mL}$, and B27 (B27 and medium at a 1:50 volume ratio) and agitated using a benchtop roller (Wheaton, Millville, NJ). The medium was changed every $2-3$ days.

\section{Synthesis and characterization of PEG-PCL diblock copolymers}

PEG-PCL diblock copolymers were synthesized as previously described, with minor modifications. ${ }^{13,18}$ Briefly, ring-opening polymerization of $\varepsilon$-caprolactone in $\mathrm{CH}_{2} \mathrm{CL}_{2}$ was induced using $\mathrm{mPEG}$ and calcium ammoniate as the macroinitiator and catalyst, respectively. Precipitates were obtained by adding cold methanol, collected by filtration, and vacuum dried at $40^{\circ} \mathrm{C}$. The molecular weight of the diblock copolymer was determined by ${ }^{1} \mathrm{H}$ nuclear magnetic resonance spectroscopy using the intensity of the terminal methoxy proton signal of $\mathrm{mPEG}$ at Q $3.39 \mathrm{ppm}$ and the methylene proton signal of polycaprolactone at $2.31 \mathrm{ppm}$. The weight ratio of the repeated PEG-PCL units was calculated to be 7.08 from the integral values of characteristic peaks, and the molecular weight was determined to be 19,278 Da. A similar molecular weight was also obtained by gel permeation chromatography.

\section{Preparation of composite doxorubicin- loaded micelles}

As we have previously reported, ${ }^{13}$ polymeric micelles containing doxorubicin were prepared according to the solvent evaporation method. Briefly, $3 \mathrm{mg}$ doxorubicin was dissolved in $2 \mathrm{~mL}$ of $\mathrm{CH}_{2} \mathrm{Cl}_{2}$ in the presence of a triple molar ratio of triethylamine and stirred at $500 \mathrm{rpm}$ for 4 hours at room temperature. PEG-poly(lactic-co-glycolic acid) (PLGA) and Pluronic 105 at a 1:5 ratio were then added and vortexed until completely dissolved. Following this, the organic solution containing the drug and polymer was added to $20 \mathrm{~mL}$ of distilled water with sonication. The mixture was stirred vigorously (10,000 rpm) for one hour, and then stirred slowly (200 rpm) overnight. Residual $\mathrm{CH}_{2} \mathrm{Cl}_{2}$ was removed by a rotary evaporator. The micelle solution was concentrated to $5 \mathrm{~mL}$ and filtered (using a $0.22 \mu \mathrm{m}$ pore size) to eliminate polymer and doxorubicin aggregates. All procedures were carried out with protection from light.

\section{Micelle characterization}

The morphology of the micelles was observed under a Morgagni 268 D transmission electron microscope (FEI, Eindhoven, The Netherlands) and particle size was measured by dynamic light scattering (Malvern Nano ZS, Malvern, Worcestershire, UK). The unloaded drug was separated by a filtration membrane (Millipore, Bedford, MA), and analytical high-performance liquid chromatography (Agilent 1100 system, Santa Clara, CA) was used to quantify the amount of doxorubicin. Drug entrapment efficiency was calculated as the amount of doxorubicin entrapped in the micelle with respect to total micellar doxorubicin.

\section{Cytotoxicity assay}

A549 cells were maintained in RPMI 1640 medium supplemented with $10 \%$ fetal bovine serum and $1 \%$ penicillin-streptomycin at $37^{\circ} \mathrm{C}$ and in a humidified $5 \% \mathrm{CO}_{2}$ atmosphere. A549 cells were seeded in 96-well plates at a density of 3500 cells/well and allowed to grow overnight. On the following day, a set concentration of the various doxorubicin formulations was added into the culture medium. After 72 hours of incubation, the cytotoxic activity of doxorubicin was then evaluated using a standard MTT assay. Blank composite micelles were also tested for cell inhibition by MTT. All experiments were performed in triplicate. The results were calculated using the following equation:

Percentage cytotoxicity $=[$ Absorbency $($ control group $)$

- Absorbency (experiment group)]/Absorbency (control group) $\times 100 \%$

\section{Fluorescent high-performance liquid chromatography}

For evaluation of the accumulation and release of doxorubicin in A549 cells, a cell suspension containing the same concentration $(2.5 \mu \mathrm{g} / \mathrm{mL})$ of free doxorubicin or composite doxorubicin-loaded micelles, was seeded at a density of $1 \times 10^{6}$ cells $/ \mathrm{mL}$ in $2 \mathrm{~mL}$ of growth medium. To measure doxorubicin accumulation, cells were incubated for 4 hours. To detect released doxorubicin, cells were incubated with 
doxorubicin for 4 hours, then the medium was removed and the cells were washed three times with phosphate-buffered saline. Fresh complete medium was then added and the cells were harvested one hour and 2.5 hours later. At each time point for measurement of accumulation and release of doxorubicin, the medium was removed, the cells were washed three times using ice-cold phosphate-buffered saline to remove noninternalized doxorubicin, and were subsequently resuspended in water $(1 \mathrm{~mL})$ and treated by probe-type ultrasonication 20 times $\left(4^{\circ} \mathrm{C}, 200 \mathrm{~W}\right.$, active every 2 seconds for a duration of 5 seconds) to obtain the cell lysate. The lysate was centrifuged at 10,000 rpm for 5 minutes in a microcentrifuge (Hettich Zentrifugen, D-78532, Tuttlingen, Germany) to remove the cellular debris. The supernatant was analyzed by high-performance liquid chromatography (Agilent 1100) equipped with a multiwavelength fluorescence detector (excitation $488 \mathrm{~nm}$ and emission $570 \mathrm{~nm}$ ). Calibration curves of known concentrations of doxorubicin in water were used for quantification. The chromatographic column used was an Agilent Zorbax Extend C18 (4.6×250 mm, $5 \mu \mathrm{m})$, and the mobile phase used was acetonitrile/0.015 $\mathrm{M} \mathrm{NaH}_{2} \mathrm{PO}_{4}$ solution ( $\mathrm{pH} 4.5,30: 70)$, with the flow rate kept at $1.0 \mathrm{~mL}$ per minute.

\section{Confocal laser scanning microscopy}

Three-dimensional A549 multicellular spheroids were plated into 96-well culture dishes (Corning, NY) and identical concentrations of either free doxorubicin or composite doxorubicin-loaded micelles were added to the multicellular spheroids and incubated for 4 hours. The medium was then removed, the multicellular spheroids were washed with cold phosphate-buffered saline, and fresh complete medium was then added and the intensity of doxorubicin fluorescence and penetration was examined under a confocal laser scanning microscope (Zeiss LSM 510, Zurich, Switzerland) to determine the accumulation and release of the drug. Doxorubicin excitation and emission occurred at $485 \mathrm{~nm}$ and $595 \mathrm{~nm}$, respectively.

\section{Flow cytometry analysis}

The intensity of doxorubicin fluorescence in the multicellular spheroids was determined by flow cytometry (FACSCalibur, Becton-Dickinson, Franklin Lakes, NJ). A549 spheroids were trypsinized, blown into a single cell suspension, and seeded into 24-well culture dishes at a density of $5 \times 10^{5}$ cells/well. The same concentration $(2.5 \mu \mathrm{g} / \mathrm{mL})$ of composite doxorubicin-loaded micelles or free doxorubicin was added to the culture medium, taking the efficiency of doxorubicin loading into composite micelles into account. Cells were harvested after 2 and 3 hours to measure accumulation of doxorubicin. To detect the amount of doxorubicin released, the cells were incubated with doxorubicin for 3 hours, the medium was then removed, and the cells were washed three times with phosphate-buffered saline. Fresh complete medium was then added, and the cells were harvested 2 hours and 4 hours later. At each time point, the medium was removed, the cells were washed three times with phosphate-buffered saline, and incorporation of doxorubicin was determined by fluorescence (488 $\mathrm{nm}$ excitation and $585 \mathrm{~nm}$ emission). Data were analyzed using FlowJo7.6 software.

\section{Cell irradiation}

Cell irradiation was performed using a medical linear accelerator (Primus-M, Siemens, Germany). The radiation field, dose rate, and source-to-cell distance were $40 \mathrm{~cm} \times 40 \mathrm{~cm}$, 2 Gy per minute, and $100 \mathrm{~cm}$, respectively.

\section{lonizing radiation and colony-forming assay}

A549 multicellular spheroids were exposed to $2.5 \mu \mathrm{g} / \mathrm{mL}$ blank composite micelles, doxorubicin composite micelles (MIX), or free doxorubicin for 4 hours. The multicellular spheroids were then trypsinized, counted, and seeded into 6-well culture dishes, irradiated (at 0-6 Gy), and incubated for a further 10 days. After this time, the cell colonies were washed with cold phosphate-buffered saline, fixed with $75 \%$ methanol, and stained with $0.5 \%$ crystal violet. Colonies containing more than 50 cells were counted using a dissecting microscope and cell survival curves were generated using GraphPad Prism 5.0 (GraphPad Software Inc, La Jolla, CA) based on the multitarget/single-hit model:

$$
S=1-\left(1-e^{-D / D O}\right)^{N}(\mathrm{~L}-\mathrm{Q})
$$

and $\mathrm{D}_{0}$ was then calculated.

\section{Cell migration}

Multicellular spheroids with a diameter of approximately $250 \mu \mathrm{m}$ were chosen for these experiments because multicellular spheroids of this size do not undergo central necrosis and contain very few hypoxic cells. ${ }^{28}$ Each treatment group (sham, irradiation alone, blank composite micelle + irradiation, free doxorubicin + irradiation, and composite doxorubicin-loaded micelle + irradiation) consisted of 30 individual A549 multicellular spheroids in five independent replicate experiments. The multicellular spheroids were incubated with or without the drug for 4 hours, the drugs 
were then removed, the multicellular spheroids were washed twice with phosphate-buffered saline, and fresh complete medium was added. The individual multicellular spheroids were then plated into 6-well culture dishes without base-coated agarose. Under these conditions, the multicellular spheroids disassembled and released cells which migrated away from the central point. The area covered by migrated cells was measured in $\mathrm{mm}^{2}$ every 24 hours for 4 days, and the migration capability was estimated by the total area covered by the cells. The spheroid migration rate was calculated using the formula:

[1 - (The mean spheroid migration area of test group/

The mean spheroid migration area of control group)] $\times 100 \%$.

\section{Proliferation assay}

Multicellular spheroids with diameters of approximately $250 \mathrm{~mm}$ were plated into 6 -well culture dishes coated with 5\% agarose. Each treatment group (sham, irradiation alone, blank composite micelle + irradiation, free doxorubicin + irradiation, and composite doxorubicin-loaded micelle + irradiation) consisted of 30 individual A549 multicellular spheroids in five independent replicate experiments. To study the response to treatment, the multicellular spheroids were observed for 9 days, with monitoring of their growth every second day. Spheroid volume was calculated using the equation:

$$
\mathrm{V}=(4 / 3) \pi \mathrm{R}^{3} \quad \mathrm{R}=(\mathrm{D} 1+\mathrm{D} 2) / 4
$$

where D1 and D2 are the maximal diameters of multicellular spheroids measured in the rectangular direction. A statistical comparison of the original data was performed using an unpaired sample $t$-test following analysis of variance. $P$ values $<0.05$ were considered to be statistically significant. The proliferation rate of the spheroids was calculated using the formula:

[1 - (Mean spheroid proliferation volume of test group/

Mean spheroid proliferation volume of control group)] $\times 100 \%$.

\section{Results}

\section{Properties of composite doxorubicin- loaded micelles}

Transmission electron microscopy revealed that the composite PEG-PCL/P105 micelles were spherical, with a uniform particle size distribution of approximately $20 \mathrm{~nm}$, calculated using the mean diameter (number distribution). The entrapment efficiency of doxorubicin was $56.7 \% \pm 1.8 \%$. The critical micelle concentration, ie, the concentration of surfactants above which micelles form and for which almost all additional surfactants are incorporated into micelles, was determined by the fluorescence intensity ratio (I338/I333) using pyrene as a fluorescent probe and calculated to be $0.98 \mu \mathrm{g} / \mathrm{mL}$ and $0.92 \mu \mathrm{g} / \mathrm{mL}$ for PEG-PCL (EG/CL 7.08) micelles and Pluronic 105 micelles, respectively.

\section{Cytotoxicity of different doxorubicin in A549 cells}

The cytotoxicity of free doxorubicin, the blank composite micelles, and the composite doxorubicin-loaded micelles in A549 cells was evaluated. As shown in Figure 1, the composite doxorubicin-loaded micelles showed notable improvement of cytotoxicity compared with free doxorubicin. The blank composite micelles rarely caused cell death, indicating that any cytotoxicity was caused by doxorubicin rather than the carrier material.

\section{More cellular uptake and sustained release of doxorubicin-loaded micelles}

Internalization of composite doxorubicin-loaded micelles and free doxorubicin in the A549 tumor cells was examined by fluorescent high-pressure liquid chromatography. As shown in Figure 2, after 4 hours of incubation, envelopment of doxorubicin in the composite micelles significantly increased cellular accumulation of the drug. After treatment with composite doxorubicin-loaded micelles or free doxorubicin for 4 hours, followed by one-hour recovery and 2.5-hour recovery periods, envelopment of doxorubicin in the composite micelles produced significantly sustained cellular release of the drug.

\section{More cellular accumulation and slower release of doxorubicin-loaded micelles}

Intracellular incorporation and release of doxorubicin in A549 multicellular spheroids was measured by confocal

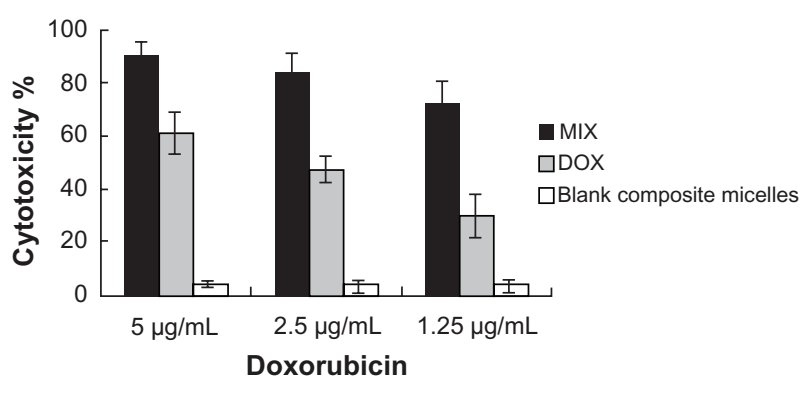

Figure I Cytotoxicity determined by MTT assay in A549 cells.

Notes: Cells were incubated with doxorubicin-loaded composite micelles, free doxorubicin, and blank composite micelles for 72 hours.

Abbreviations: DOX, free doxorubicin; MIX, composite doxorubicin-loaded micelles. 


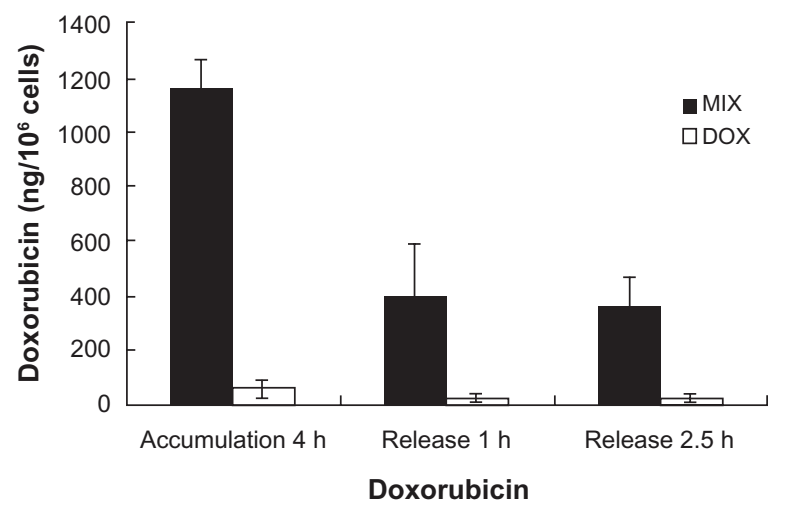

Figure 2 Intracellular doxorubicin in A549 cells.

Notes: Doxorubicin uptake into A549 tumor cells after incubation with composite doxorubicin-loaded micelles or free doxorubicin for 4 hours, then compounds removed for one hour and 2.5 hours. Accumulation 4 hours: A549 cells incubated in composite doxorubicin-loaded micelles or free doxorubicin for 4 hours; release one hour. A549 cells incubated in composite doxorubicin-loaded micelles or free DOX for 4 hours, then compounds removed for one hour; release 2.5 hours, A549 cells incubated in composite doxorubicin-loaded micelles or free doxorubicin for 4 hours, then compounds removed for 2.5 hours.

Abbreviations: DOX, free doxorubicin; MIX, composite doxorubicin-loaded micelles.

laser scanning microscopy (Figure 3). After 4 hours of incubation, A549 multicellular spheroids incubated with MIX (Figure 3B1) were more intensely fluorescent and showed deeper penetration of fluorescence than multicellular spheroids treated with free doxorubicin (Figure 3A1), which had barely detectable levels of intracellular fluorescence. Only very weak fluorescence was observed for multicellular spheroids treated with free doxorubicin for 4 hours followed by a one-hour recovery period (Figure 3A2). In contrast, multicellular spheroids treated with MIX were
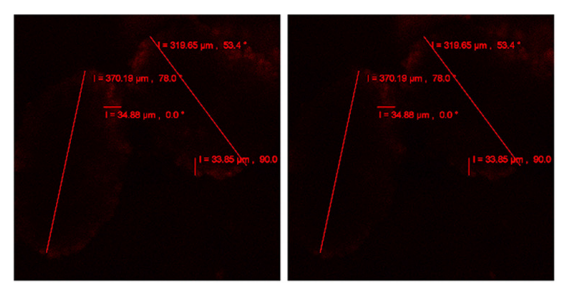

A1

A2



B1

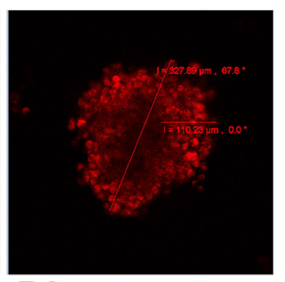

B2

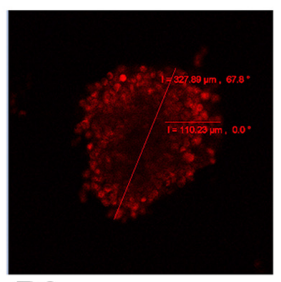

Figure 3 Confocal laser scanning microscopy of cellular incorporation and release of doxorubicin in A549 multicellular spheroids. (AI) A549 spheroid cells incubated in free doxorubicin for 4 hours. (A2) Free doxorubicin released from the spheroid for one hour. (BI) A549 spheroid cells incubated in composite doxorubicin-loaded micelles with the same doxorubicin densities for 4 hours. (B2) Composite doxorubicin-loaded micelles released from the spheroid for one hour. (B3) Composite doxorubicinloaded micelles released from the spheroid for 2.5 hours. strongly fluorescent one hour after withdrawal of the drug (Figure 3B2). Moreover, levels of fluorescence remained high at 2.5 hours (Figure 3B3).

\section{More cellular uptake and slower release from doxorubicin-loaded micelles}

Multicellular spheroid intracellular doxorubicin uptake and release by MIX and free doxorubicin was investigated by flow cytometry. As shown in Figure 4, after 2 and 3 hours, the intensity of cellular fluorescence was 14.14 and 13.61 times higher in MIX-treated multicellular spheroids than in free doxorubicin-treated multicellular spheroids for equivalent $2.5 \mu \mathrm{g} / \mathrm{mL}$ drug treatments. For multicellular spheroids incubated with MIX or free doxorubicin that achieved the same amount of doxorubicin incorporation after 3 hours of exposure, the intensity of cellular fluorescence in MIXtreated A549 cells at 2 and 4 hours following drug withdrawal was 9.53 and 14.93 times that of the free doxorubicin-treated cells, respectively (Figure 5). Overall, the quantitative results obtained by flow cytometry are in good agreement with those obtained by confocal laser scanning microscopy (Figure 3).

\section{Colony formation assays}

Cell survival curves are showed in Figure 6. $\mathrm{D}_{0} \mathrm{~s}$ was 1.466 Gy, 1.405 Gy, 1.230 Gy, and 1.020 Gy, respectively, for the groups that received irradiation only, blank composite micelles + irradiation, doxorubicin + irradiation, and MIX + irradiation, respectively. The sensitizing enhancement ratio of cells treated with equivalent amounts of free doxorubicin and doxorubicin-loaded micelles were 1.19 and 1.44, respectively $(P<0.05)$. There was no difference between the group receiving irradiation only and the group receiving blank composite micelles + irradiation.

\section{Cell migration assays}

Cell migration was determined for both treated and untreated spheroids. A significant growth reduction of $30 \%$ and $28 \%$, respectively, was seen in the irradiation only group and the blank composite micelles + irradiation group after 4 days. There was no statistically significant difference between the irradiation only group and the blank composite micelles + irradiation group. However, combined treatment with equivalent concentrations of free doxorubicin or composite doxorubicin-loaded micelles had a significant additive effect on growth reduction of $46 \%$ and $61 \%$, respectively (Figure 7). 


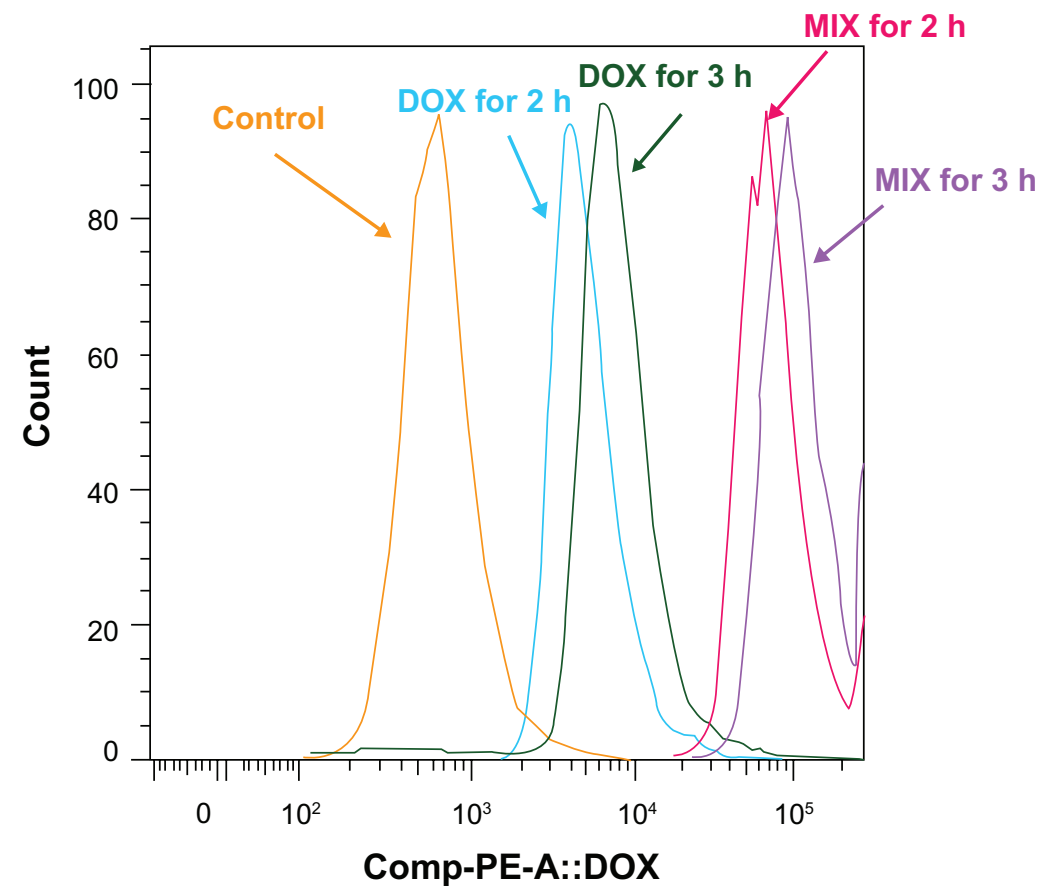

Figure 4 Flow cytometric histograms of A549 spheroid cells incubated with composite doxorubicin-loaded micelles or free doxorubicin with the same doxorubicin densities for 2 hours and 3 hours. DOX for 2 hours, multicellular spheroids incubated in free doxorubicin for 2 hours; DOX for 3 hours, multicellular spheroids incubated in free doxorubicin for 3 hours; MIX for 2 hours, multicellular spheroids incubated in MIX for 2 hours; MIX for 3 hours, multicellular spheroids incubated in MIX for 3 hours. Abbreviations: DOX, free doxorubicin; MIX, composite doxorubicin-loaded micelles.

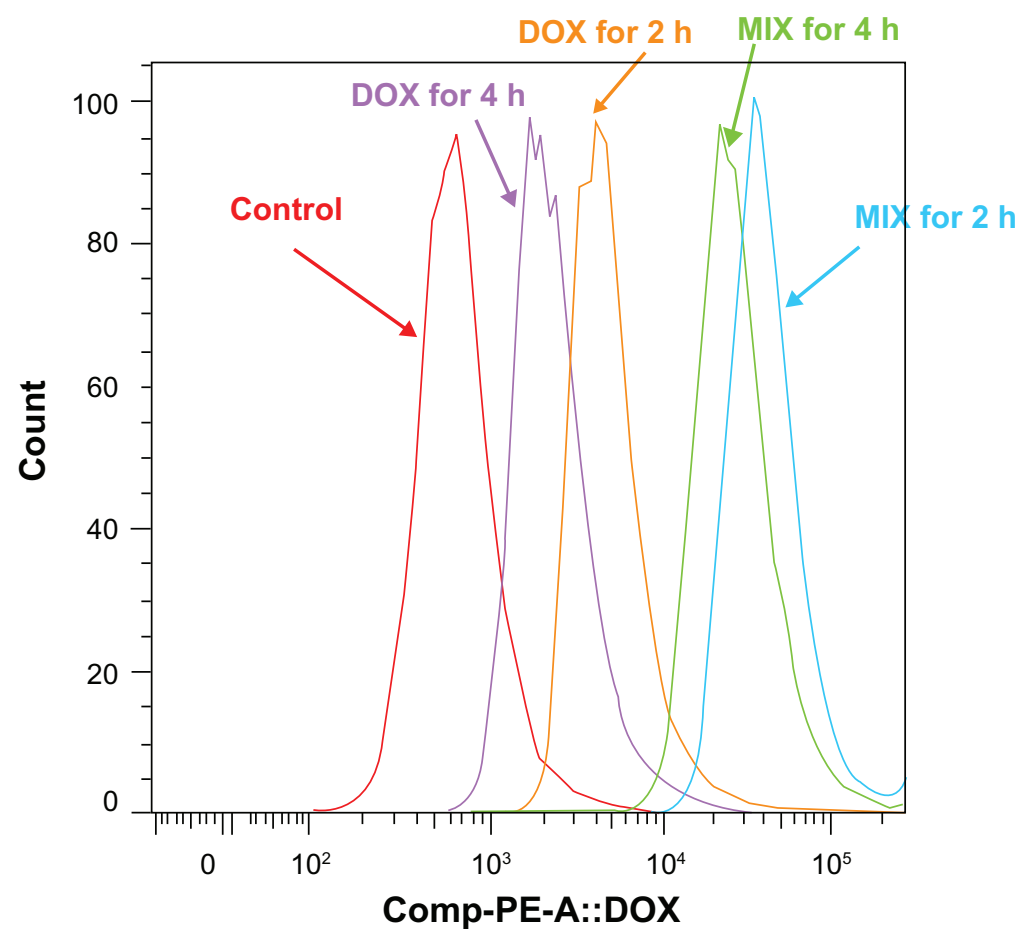

Figure 5 Flow cytometric histograms of multicellular spheroids incubated with MIX or free doxorubicin that achieved the same doxorubicin incorporation after 3 hours of exposure, at 2 and 4 hours following drug withdrawal.

Notes: DOX for 2 hours, multicellular spheroids incubated in free doxorubicin for 3 hours, then compounds removed for 2 hours; DOX for 4 hours, multicellular spheroids incubated in free doxorubicin for 3 hours, then compounds removed for 4 hours; MIX for 2 hours, multicellular spheroids incubated in MIX for 3 hours, then compounds removed for 2 hours; MIX for 4 hours, multicellular spheroids incubated in MIX for 3 hours, then compounds removed for 4 hours.

Abbreviations: DOX, free doxorubicin; MIX, composite doxorubicin-loaded micelles. 


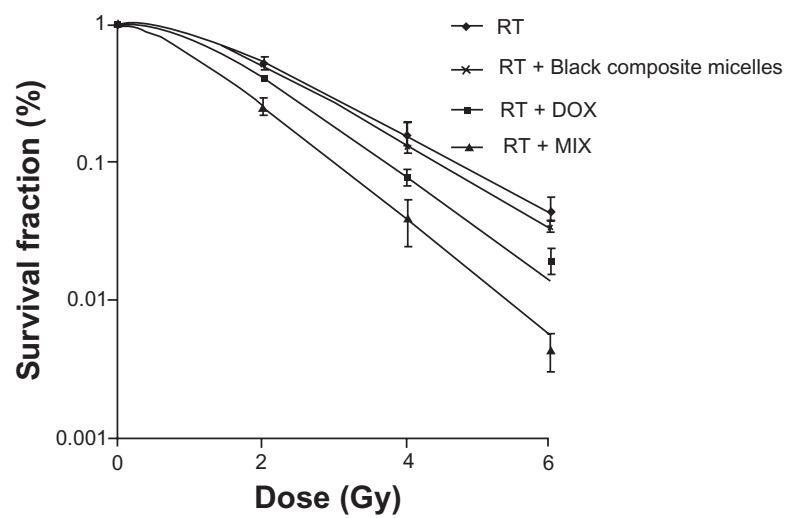

Figure 6 Cell survival curves after treatments with radiation alone or combined with $2.5 \mu \mathrm{g} / \mathrm{mL}$ blank composite micelles (radiation alone + blank composite micelles) or doxorubicin (radiation alone + doxorubicin) or doxorubicin-loaded composite micelle (radiation alone + MIX, $P<0.05$ for radiation alone + MIX versus radiation alone). Abbreviations: DOX, free doxorubicin; MIX, composite doxorubicin-loaded micelles; $\mathrm{RT}$, radiation alone; Gy, gray.

\section{Cell proliferation assays}

The proliferation capacity of spheroids treated with equivalent concentrations of doxorubicin or MIX was determined. Following irradiation alone and blank composite micelles + irradiation, the spheroids showed significant growth reduction of $44 \%$ and $47 \%$, respectively, after 9 days. There was no difference between the irradiation only group and the blank composite micelles + irradiation group. However, following combined treatment, the spheroids showed significantly increased growth reduction of $58 \%$ (for radiation + free doxorubicin) and 77\% (for radiation + MIX) after nine days (Figure 8).

\section{Discussion}

Radiotherapy is very effective for local control of tumors, but its curative potential is limited by the intrinsic radioresistance

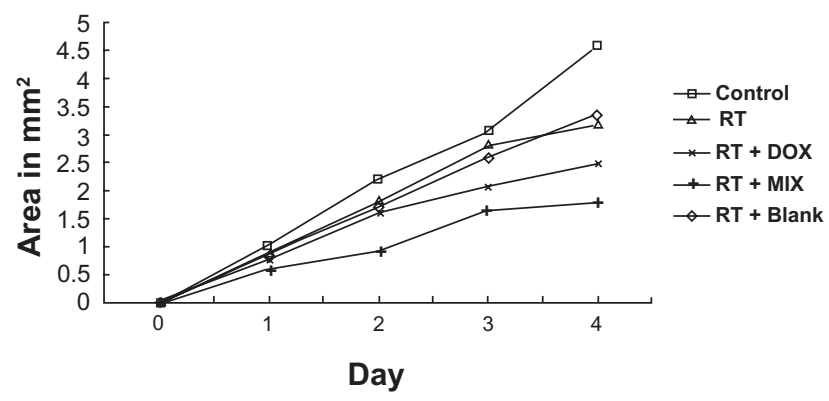

Figure 7 Tumor cell migration from spheroids following irradiation and combination treatment (radiation alone + blank composite micelles, radiation alone + doxorubicin, radiation alone + MIX). Mean area of tumor cell migration $\left(\mathrm{mm}^{2}\right)$.

Notes: Each treatment group (sham, irradiation alone, blank composite micelle + irradiation, free doxorubicin + irradiation, composite doxorubicin-loaded micelle + irradiation) consists of $30 \mathrm{~A} 549$ spheroids in five independent experiments. Changes at day 4 were significant $(P<0.05)$.

Abbreviations: DOX, free doxorubicin; MIX, composite doxorubicin-loaded micelles; $\mathrm{RT}$, radiation alone.

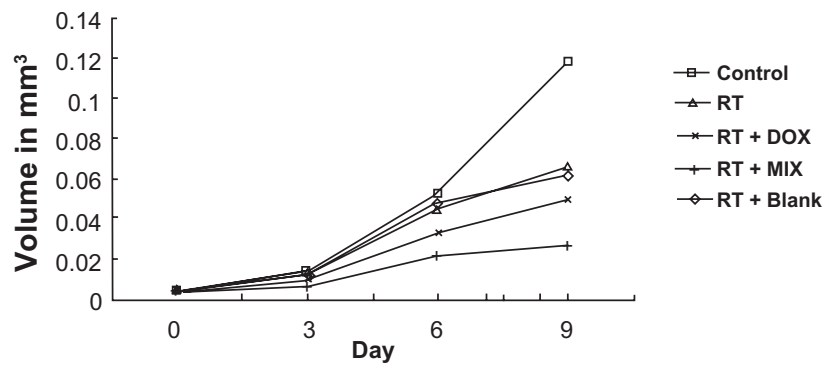

Figure 8 Spheroid proliferation following single mode and combinational treatment. Notes: Each treatment group (sham, irradiation alone, blank composite micelle + irradiation, free doxorubicin + irradiation, composite doxorubicin-loaded micelle + irradiation) consists of $30 \mathrm{~A} 549$ spheroids in five independent experiments. Illustration of mean volume growth of spheroids following irradiation and irradiation alone + blank composite micelles, irradiation alone + doxorubicin, irradiation alone + MIX (composite doxorubicin-loaded micelles). A significant reduction in spheroid volume was observed following irradiation alone + MIX on day $9(P<0.05)$.

Abbreviations: DOX, free doxorubicin; MIX, composite doxorubicin-loaded micelles; $\mathrm{RT}$, radiation alone.

of most malignant tumor cells, which often leads to treatment failure. New strategies, such as developing radiosensitizers, are clinically important and have become an important research focus.

Doxorubicin is a tetracycline antibiotic commonly used in the treatment of cancer ${ }^{19}$ and induces DNA damage by inhibition of topoisomerase II and free radical generation as an anticancer mechanism..$^{20}$ Doxorubicin can also bind covalently to DNA, leading to formation of doxorubicinDNA adducts and interstrand cross-links. For these reasons, doxorubicin is the antitumor drug most widely used for enhancement of radiotherapy. The mechanism of doxorubicinmediated radiosensitization may include induction of tumor cell apoptosis, and an increase in both sublethal radiationinduced damage and DNA repair processes.

However, in addition to its low solubility in water, doxorubicin has severe side effects, including acute toxicity to normal tissue, and its therapeutic effects can be minimized by the inherent multidrug resistance of many tumor cells. ${ }^{21} \mathrm{To}$ reduce the acute toxicity of free doxorubicin and to improve its therapeutic efficacy, various polymeric micelle systems have been designed as delivery vehicles. ${ }^{22-24}$ Studies have shown that cellular uptake of doxorubicin-loaded micelles and free doxorubicin are very different, ie, doxorubicin micelles are transported into cells by endocytosis, whereas free doxorubicin enters cells by simple diffusion. ${ }^{18}$

In this study, we prepared composite PEG-PCL and P105 micelles using the solvent evaporation method to serve as carriers for doxorubicin. This method produces a relatively small particle size distribution, due to the low molecular weight and high hydrophilicity of P105. We successfully established three-dimensional A549 multicellular spheroids 
that are a more physiologically relevant model of lung cancer than monolayer cells, because they resemble the architecture of in vivo tumors ${ }^{17,25,26}$ and can recapitulate in vivo processes which may affect drug performance, such as cell-cell contact, variations in the cell cycle, altered metabolism, and diffusion of nutrients, oxygen, or drugs. ${ }^{17,25,27,28}$ Multicellular spheroids are relatively easy to produce and maintain in culture, and treatment-related changes in spheroid growth kinetics and tumor cell outgrowth are established and reproducible endpoints. ${ }^{17,25,29}$ As a consequence of their particular architectural characteristics, multicellular spheroids have been demonstrated to be extremely useful for testing radiotherapy protocols. ${ }^{30}$

We studied the doxorubicin sensitization mechanism in a number of experiments. First, we used MTT to study the cytotoxicity of different doxorubicin formulations in A549 cells. As shown in Figure 1, composite doxorubicinloaded micelles showed notable improvement in cytotoxicity compared with free doxorubicin. Blank composite micelles rarely caused cell death, indicating that cytotoxicity was caused by doxorubicin rather than by the carrier material. Internalization of composite doxorubicin-loaded micelles and free doxorubicin into A549 tumor cells was then examined by fluorescent high-performance liquid chromatography. As shown in Figure 2, after 4 hours of incubation, envelopment of doxorubicin in the composite micelles significantly increased intracellular accumulation of the drug. After treatment with composite doxorubicin-loaded micelles or free doxorubicin for 4 hours, followed by a one-hour recovery or 2.5-hour recovery period, envelopment of doxorubicin in the composite micelles sustained cellular release of the drug in a significant manner.

We also undertook a series of experiments in A549 multicellular spheroids, in which we investigated accumulation and efflux of doxorubicin by confocal laser scanning microscopy. As shown in Figure 3, after 4 hours, A549 multicellular spheroids incubated with MIX showed a greater fluorescent intensity and deeper penetration than those treated with free doxorubicin, in which intracellular fluorescence was barely detectable. In contrast, high levels of fluorescence were still detectable in MIX-treated spheroids 2.5 hours after drug withdrawal, indicating that use of composite doxorubicinloaded micelles leads to improved cellular retention of doxorubicin.

By measuring the intensity of fluorescence and depth of doxorubicin penetration into multicellular spheroids, we found that treatment with doxorubicin-loaded micelles led to higher intracellular accumulation and deeper penetration than that obtained for free doxorubicin. In addition, the use of micelle carriers led to a longer doxorubicin retention time in multicellular spheroids. Our previous study ${ }^{13}$ also showed rapid efflux of free doxorubicin from K562/ADR cells. However, unlike doxorubicin in solution, uptake of composite doxorubicin-loaded PEG-PCL/P105 micelles occurs via endocytosis. Micelles enter cells slowly, avoid being pumped out, and continue to accumulate around the cell nucleus. Similar results were obtained by Shuai et al, ${ }^{18}$ who reported that free doxorubicin was rapidly pumped out of cells, but that micelle carriers inhibited doxorubicin efflux and improved retention in cells.

Furthermore, quantitative flow cytometry studies verified that, for equivalent doxorubicin concentrations, composite doxorubicin-loaded micelles significantly enhanced cellular doxorubicin accumulation and inhibited doxorubicin release. Doxorubicin uptake in MIX-treated multicellular spheroids was 14.14 and 13.61 times greater than in multicellular spheroids treated with free doxorubicin after 2 hours and 3 hours, respectively. In addition, doxorubicin retention was 9.53 and 14.93 times greater in MIX-treated multicellular spheroids than in multicellular spheroids treated with an equivalent concentration of free doxorubicin 2 and 4 hours, respectively, after drug withdrawal. Overall, our quantitative results (shown in Figures 4 and 5) agree with those obtained by confocal laser scanning microscopy (Figure 3), and suggest that doxorubicin binding to the hydrophobic core of the micelle may prevent its efflux.

Colony-forming assays also demonstrated that composite doxorubicin-loaded micelles are radiosensitive, as shown by significantly reduced survival of cells treated by radiation + composite micelles compared with those treated with radiation + free doxorubicin, radiation alone, or radiation + blank composite micelles. The surviving fraction of cells treated with MIX + radiation was significantly lower than for cells subject to irradiation alone or radiation + blank composite micelles $(P<0.05)$. However, there was no statistically significant difference in cell death between cells treated with a combination of free doxorubicin + irradiation relative to irradiation alone or radiation + blank composite micelles.

Finally, the cellular migration area and growth capability of multicellular spheroids confirmed the increased radiosensitivity of cells treated with composite doxorubicin-loaded micelles compared with those treated with free doxorubicin. In short, we successfully increased the radiosensitivity of A549 multicellular spheroids using composite micelles loaded with doxorubicin. At equivalent drug concentrations, composite doxorubicin-loaded PEG-PCL/P105 micelles 
significantly enhanced the accumulation of doxorubicin in multicellular spheroids and inhibited release of doxorubicin from multicellular spheroids, thus achieving improved radiosensitivity. Preventing a burst of doxorubicin release may avoid excessive damage to normal tissue, minimizing cytotoxic effects. Therefore, this study provides a novel approach to improving radiosensitivity of tumor cells, and may achieve an improved therapeutic outcome for cancers that cannot be well controlled by traditional radiotherapy. Clinical application of this research may reduce the side effects of conventional cancer therapies, and thereby improve patient compliance.

In summary, composite doxorubicin-loaded micelles consisting of PEG-PCL/Pluronic P105 can significantly enhance doxorubicin radiosensitivity in lung cancer multicellular spheroids and provide a novel approach to lung cancer treatment. Further similar investigations should be undertaken in other tumor models. Further, combined treatment with micelle-encapsulated doxorubicin and radiation therefore warrants investigation in clinical trials as a potential anticancer strategy.

\section{Acknowledgments}

This work was supported by the Foundation of Zhejiang Educational Committee (Y200909276), the National Natural Science Foundation of China (30970863), and the National Natural Science Foundation of China (81071823).

\section{Disclosure}

The authors report no conflicts of interest in this work.

\section{References}

1. Jemal A, Siegel R, Xu J, Ward E. Cancer statistics, 2010. CA Cancer J Clin. 2010;60:277-300.

2. Collins LG, Haines C, Perkel R, Enck RE. Lung cancer: diagnosis and management. Am Fam Physician. 2007;75:56-63.

3. Zhang X, Yang H, Gu K, Chen J, Rui M, Jiang GL. In vitro and in vivo study of a nanoliposomal cisplatin as a radiosensitizer. Int $J$ Nanomedicine. 2011;6:437-444.

4. Yu J, Liu F, Sun Z, Sun M, Sun S. The enhancement of radiosensitivity in human esophageal carcinoma cells by thalidomide and its potential mechanism. Cancer Biother Radiopharm. 2011;26:219-227.

5. Zhang XD, Guo ML, Wu HY, et al. Irradiation stability and cytotoxicity of gold nanoparticles for radiotherapy. Int J Nanomedicine. 2009;4: 165-173.

6. Kohori F, Yokoyama M, Sakai K, Okano T. Process design for efficient and controlled drug incorporation into polymeric micelle carrier systems. $J$ Control Release. 2002;78:155-163.

7. Rapoport N, Marin A, Luo Y, Prestwich GD, Muniruzzaman MD. Intracellular uptake and trafficking of Pluronic micelles in drug-sensitive and MDR cells: effect on the intracellular drug localization. J Pharm Sci. 2002;91:157-170.

8. Khonkarn R, Mankhetkorn S, Hennink WE, Okonogi S. PEG-OCL micelles for quercetin solubilization and inhibition of cancer cell growth. Eur J Pharm Biopharm. 2011;79:268-275.
9. An L, Wang Y, Liu X, et al. Block ionomer complex micelles based on the self-assembly of poly(ethylene glycol)-block-poly(acrylic acid) and $\mathrm{CdCl}(2)$ for anti-tumor drug delivery. Chem Pharm Bull (Tokyo). 2011;59:559-563.

10. Qiu L, Zhang L, Zheng C, Wang R. Improving physicochemical properties and doxorubicin cytotoxicity of novel polymeric micelles by poly( $\varepsilon$-caprolactone) segments. J Pharm Sci. 2011;100: 2430-2442.

11. Kataoka K, Harada A, Nagasaki Y. Block copolymer micelles for drug delivery: design, characterization and biological significance. Adv Drug Deliv Rev. 2001;47:113-131.

12. Diao YY, Han M, Ding PT, Chen DW, Gao JQ. DOX-loaded PEG-PLGA and Pluronic copolymer composite micelles enhances cytotoxicity and the intracellular accumulation of drug in DOX-resistant tumor cells. Pharmazie. 2010;65:356-358.

13. Han M, Diao YY, Jiang HL, et al. Molecular mechanism study of chemosensitization of doxorubicin-resistant human myelogenous leukemia cells induced by a composite polymer micelle. Int J Pharm. 2011;420:404-411.

14. Cetintas VB, Kucukaslan AS, Kosova B, et al. Cisplatin resistance induced by decreased apoptotic activity in non small cell lung cancer cell lines. Cell Biol Int. 2012;36:261-265.

15. Lee H, Kim S, Choi BH, et al. Hyperthermia improves therapeutic efficacy of doxorubicin carried by mesoporous silica nanocontainers in human lung cancer cells. Int J Hyperthermia. 2011;27:698-707.

16. Lim M, Chuong CM, Roy-Burman P. PI3K, ERK signaling in BMP7induced epithelial-mesenchymal transition (EMT) of PC-3 prostate cancer cells in 2- and 3-dimensional cultures. Horm Cancer. 2011; 2:298-309.

17. Fehlauer F, Muench M, Richter E, Rades D. The inhibition of proliferation and migration of glioma spheroids exposed to temozolomide is less than additive if combined with irradiation. Oncol Rep. 2007; 17:941-945.

18. Shuai X, Ai H, Nasongkla N, Kim S, Gao J. Micellar carriers based on block copolymers of poly(epsilon-caprolactone) and poly(ethylene glycol) for doxorubicin delivery. J Control Release. 2004;98: 415-426.

19. Kievit FM, Wang FY, Fang C, et al. Doxorubicin loaded iron oxide nanoparticles overcome multidrug resistance in cancer in vitro. J Control Release. 2011;152:76-83.

20. Mizutani H, Tada-Oikawa S, Hiraku Y, Kojima M, Kawanishi S. Mechanism of apoptosis induced by doxorubicin through the generation of hydrogen peroxide. Life Sci. 2005;76:1439-1453.

21. Yoo HS, Park TG. Biodegradable polymeric micelles composed of doxorubicin conjugated PLGA-PEG block copolymer. Control Release. 2001;70:63-70.

22. Gao ZG, Tian L, Hu J, Park IS, Bae YH. Prevention of metastasis in a 4T1 murine breast cancer model by doxorubicin carried by folate conjugated pH sensitive polymeric micelles. J Control Release. 2011; 152:84-89.

23. Kim D, Lee ES, Oh KT, Gao ZG, Bae YH. Doxorubicin-loaded polymeric micelle overcomes multidrug resistance of cancer by doubletargeting folate receptor and early endosomal pH. Small. 2008;4: 2043-2050.

24. Inoue T, Yamashita Y, Nishihara M, et al. Therapeutic efficacy of a polymeric micellar doxorubicin infused by convection-enhanced delivery against intracranial 9L brain tumor models. Neuro Oncol. 2009;11:151-157.

25. Fehlauer F, Muench M, Rades D, et al. Effects of irradiation and cisplatin on human glioma spheroids: inhibition of cell proliferation and cell migration. J Cancer Res Clin Oncol. 2005;131:723-732.

26. Fehlauer F, Stalpers LJ, Panayiotides J, et al. Effect of single dose irradiation on human glioblastoma spheroids in vitro. Oncol Rep. 2004; 11 : $477-485$.

27. Walenta S, Doetsch J, Mueller-Klieser W, Kunz-Schughart LA. Metabolic imaging in multicellular spheroids of oncogene-transfected fibroblasts. J Histochem Cytochem. 2000;48:509-522. 
28. Sminia P, Acker H, Eikesdal HP, et al. Oxygenation and response to irradiation of organotypic multicellular spheroids of human glioma Anticancer Res. 2003;23:1461-1466.

29. Gliemroth J, Zulewski H, Arnold H, Terzis AJ. Migration, proliferation, and invasion of human glioma cells following treatment with simvastatin. Neurosurg Rev. 2003;26:117-124.
30. Santini MT, Rainaldi G, Indovina PL. Multicellular tumour spheroids in radiation biology. Int J Radiat Biol. 1999;75:787-799.

International Journal of Nanomedicine

\section{Publish your work in this journal}

The International Journal of Nanomedicine is an international, peerreviewed journal focusing on the application of nanotechnology in diagnostics, therapeutics, and drug delivery systems throughou the biomedical field. This journal is indexed on PubMed Central,

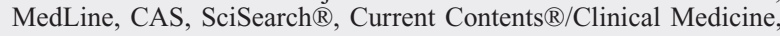

Journal Citation Reports/Science Edition, EMBase, Scopus and the Elsevier Bibliographic databases. The manuscript management system is completely online and includes a very quick and fair peer-review system, which is all easy to use. Visit http://www.dovepress.com/ testimonials.php to read real quotes from published authors.

Submit your manuscript here: http://www.dovepress.com/international-journal-of-nanomedicine-journal 